\title{
Manipulation of $\mathrm{C}_{60}$ islands on the rutile $\mathrm{TiO}_{2}$ (110) surface using noncontact atomic force microscopy
}

\author{
Felix Loske and Angelika Kühnle ${ }^{a}$ \\ Fachbereich Physik, Universität Osnabrück, Barbarastraße 7, 49076 Osnabrück, Germany
}

(Received 15 May 2009; accepted 28 May 2009; published online 30 July 2009)

\begin{abstract}
Regular, almost quadratic pits were created in an island of $\mathrm{C}_{60}$ molecules on a rutile $\mathrm{TiO}_{2}(110)$ surface using noncontact atomic force microscopy at room temperature. Upon gradually approaching the scanning tip toward the surface, the interaction between the tip and the $\mathrm{C}_{60}$ island was increased until manipulation was achieved. Analyzing the manipulation process unambiguously revealed that the manipulation was performed in the repulsive regime. Retracting the tip allowed for reproducible imaging the $\mathrm{C}_{60}$ island after the manipulation process. Moreover, whole islands could be reshaped or even removed when scanning with appropriate scanning parameters. (C) 2009 American Institute of Physics. [DOI: 10.1063/1.3184784]
\end{abstract}

Long-term objective of investigating molecules on surfaces is to verify the applicability of creating materials with improved properties and functions at the nanoscale. ${ }^{1}$ A very appealing motivation is to adopt molecules as building blocks for nanoelectronics, since miniaturization of electronics by conventional lithographic methods is approaching its technical limits. ${ }^{1,2}$ To achieve ordered structures, different strategies are open to explore. The surface may be templated for a steered assembly of the molecules ${ }^{3,4}$ and exploiting molecular self-assembly is considered as a very promising strategy. ${ }^{5-7}$ However, employing parallel and autonomous techniques such as self-assembly in combination with direct patterning methods such as manipulation might allow for creating an even broader variety of structures. The most direct fabrication method of nanostructures is tip-induced manipulation, ${ }^{8}$ since the tip is controllable at the subnanometer scale.

Atomic and molecular manipulation has been studied extensively on metal surfaces using scanning tunneling microscopy (STM) and a rather comprehensive understanding of the manipulation process has been achieved. ${ }^{9-13}$ Using noncontact atomic force microscopy (NC-AFM), ${ }^{14}$ however, controlled manipulation of single atoms and atom size defects has been achieved in very few cases only. Compared to manipulation using the STM, only limited understanding has been gained so far based on experimental data ${ }^{15-22}$ and theoretical calculations. $^{23-25}$ Lateral manipulation can be triggered by lowering the energy barriers for hopping processes due to attractive interactions between tip and surface adatoms ${ }^{17,23}$ or due to repulsive interaction between the tip and surface adsorbates/atoms. ${ }^{19}$ Like in STM, pulling ${ }^{26}$ as well as pushing ${ }^{20}$ manipulation mechanisms were proposed. In contrast with previous manipulation experiments using NC-AFM, we demonstrate well-controlled manipulation of large molecules.

In this work, the system of $\mathrm{C}_{60}$ molecules on the rutile $\mathrm{TiO}_{2}$ (110) surface was investigated with NC-AFM at room temperature. ${ }^{27,28}$ The $\mathrm{C}_{60}$ fullerene ${ }^{29}$ is a promising candidate for molecular electronics due to its intrinsic amplifier effect. $^{30,31}$ Moreover, considerations have been made about an application of $\mathrm{C}_{60}$ molecules as single-molecule transistors. ${ }^{32}$ The corrugation of the $\mathrm{TiO}_{2}$ (110) surface ap-

${ }^{\text {a)} E l e c t r o n i c ~ m a i l: ~ k u e h n l e @ u o s . d e . ~}$ pears to be applicable for templating the $\mathrm{C}_{60}$ molecules in between the one-dimensional stretched troughs for an ordered assembly. ${ }^{27}$ We demonstrate the tip-induced modification of $\mathrm{C}_{60}$ islands due to clear repulsive interactions between tip and molecules. Thereby, we created pits in wellordered islands and moved domain boundaries on the islands. Additionally, altering and removal of complete $\mathrm{C}_{60}$ island is demonstrated.

Measurements were performed in an ultrahigh vacuum system (Omicron VT-AFM 25) operated in frequency modulation NC-AFM (FM NC-AFM) mode. Tip and sample preparation as well as the thermal sublimation of $\mathrm{C}_{60}$ molecules onto the surface has been described previously. ${ }^{27}$ All experiments were performed at room temperature. In the present experiments, the distance feedback was not switched off completely. ${ }^{28}$ The frequency shift setpoint serves as a measure for the tip-sample distance in our experiments. The images are displayed such that bright areas correspond to high attractive interaction while dark corresponds to less attractive or even repulsive interactions. ${ }^{33}$

On the rutile $\mathrm{TiO}_{2}$ (110) surface, $\mathrm{C}_{60}$ molecules are known to arrange in a centered rectangular superstructure with an angle of $81^{\circ} \pm 2^{\circ}$ and with the $\mathrm{C}_{60}$ molecules lying centered in the troughs between the substrate's bridging oxygen rows. The fact that islands are observed at room temperature indicates that $\mathrm{C}_{60}$ molecules are mobile at this temperature. Only rarely, individual molecules are observed on the terrace. Consequently, these molecules must be anchored to surface defects. As the density of the well-known hydroxyl defects is known to be much higher than the observed individual molecules, ${ }^{34}$ the $\mathrm{C}_{60}$ molecules are not anchored to the hydroxyl defects. Protruding molecular rows appear on the $\mathrm{C}_{60}$ islands, which are ascribed to domain boundaries originating from stacking faults. ${ }^{27}$ In Fig. 1(a), a $\mathrm{C}_{60}$ layer including such domain boundaries is displayed. When scanning on the $\mathrm{C}_{60}$ islands, we created holes into these islands by scanning at increased frequency shift setpoints. An example is shown in Fig. 1. Image (a) was recorded at a frequency shift setpoint of $-10.4 \mathrm{~Hz}$. From this point, the frequency shift setpoint was increased stepwise in the following images, resulting in contrast inversion as discussed in Ref. 28. The corresponding images can be seen in Ref. 28. Very stable imaging was possible until a setpoint of $-25.1 \mathrm{~Hz}$ was reached. At this setpoint, the tip interacts sufficiently with the 


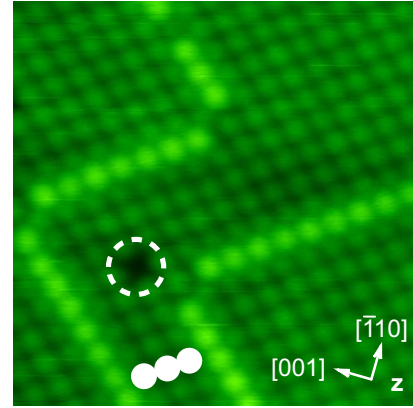

(a)

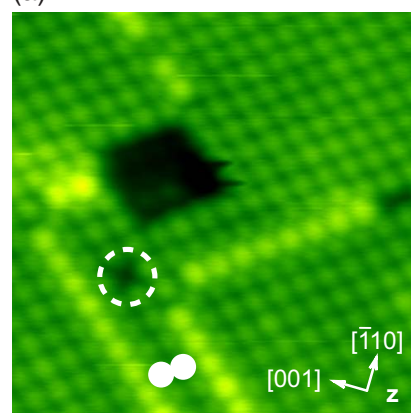

(b)
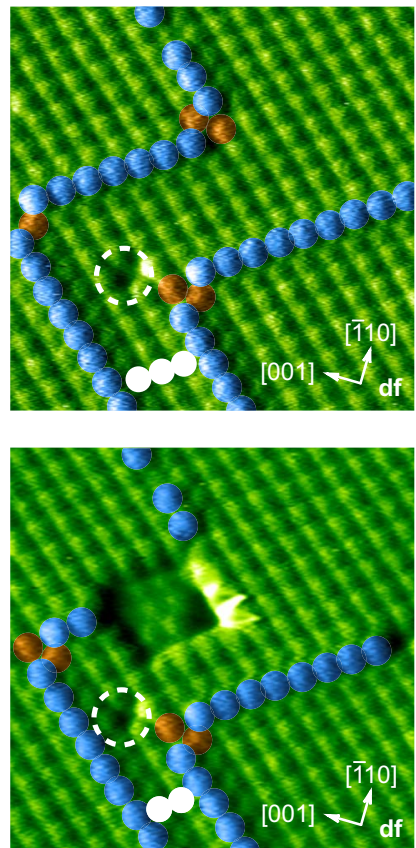

FIG. 1. (Color online) Comparison of two NC-AFM forward-scan images $\left(14 \times 14 \mathrm{~nm}^{2}\right)$ at a frequency shift setpoint of $-10.4 \mathrm{~Hz}$ before and after a stepwise increase in the frequency shift setpoint to $-25.1 \mathrm{~Hz}$ (this series can be seen in Ref. 28). Each image is shown with the topographic (left panel) and frequency shift channel (right panel). At a frequency shift setpoint of $-25.1 \mathrm{~Hz}$, the interaction between tip and $\mathrm{C}_{60}$ molecules induced an almost quadratic hole by the removal of seventeen molecules ( 12 from the plain $\mathrm{C}_{60}$ island and 5 from the upper domain boundary). Additionally, a reordering of the left domain boundary was observed. In both images, a single missing $\mathrm{C}_{60}$ molecule is marked as a reference point.

$\mathrm{C}_{60}$ molecules to remove $\mathrm{C}_{60}$ molecules from the island. In Fig. 2(a) the upper part of the image was scanned at a setpoint of $-25.1 \mathrm{~Hz}$, showing full contrast inversion (i.e., the $\mathrm{C}_{60}$ molecules appear dark while the areas in between the molecules appear bright). Near the stacking fault (see area enclosed by the dotted lines), the manipulation occurred and is analyzed in the following. Every measured scanline in this manipulation area can be seen for both forward and backward scan on the left hand side in Fig. 2. Two line scans in the forward scan reveal significant positive frequency shifts, corresponding to a repulsive interaction between tip and molecules. ${ }^{33}$ This can be related to the observed manipulation process. In the lower part of the image, the frequency shift setpoint was lowered to $-19.1 \mathrm{~Hz}$, again resulting in stable imaging. The contrast is the same again as was seen at $-19.1 \mathrm{~Hz}$ before the manipulation process. ${ }^{28}$

The situation after the manipulation [Fig. 1(b)] was recorded at the same frequency shift setpoint as the first image. Comparing both images [Figs. 1(a) and 1(b)], an almost quadratic hole was created by removal of $12 \mathrm{C}_{60}$ molecules from the plain island and 5 molecules at the upper domain boundary. In addition, a reordering of the lower left domain boundary was induced. This boundary moved one repeat distance closer to the encircled vacancy in the molecular island (indicated by three/two white circles between the domain boundaries). As the domain boundaries originate from stacking faults, ${ }^{27}$ the observed reordering must be caused by a movement of the associated stacking fault during the manipulation process. The reordering of this domain boundary is linked to a rather large mass transport, as $1+2 \times n$ molecules need to change position in order to shift the whole boundary, with $n$ being the number of $\mathrm{C}_{60}$ molecules in length on the domain boundary. Additionally, one $\mathrm{C}_{60}$ molecule needs to be removed, otherwise, the boundary cannot be moved.

In Fig. 3(a), a $\mathrm{C}_{60}$ island of $\sim 25 \mathrm{~nm}$ in length and $\sim 14 \mathrm{~nm}$ in width is scanned. In Figs. 3(a)-3(1) this island shrinks in size when scanning at frequency shift setpoints between -13.0 and $-15.0 \mathrm{~Hz}$. Consequently, in Fig. 3(d) the area reduced to about one-third of the initial size in Fig. 3(a). Figure 3(a) suggests that the island has grown on the bare terrace, however, as can be seen in Fig. 3(k), a defect in the underlying substrate surface is observed. This defect might represent an anchoring site for the island, in agreement with the previous assumption that $\mathrm{C}_{60}$ molecules require nucleation seeds such as substrate step edges or other surface defects for island growth at room temperature. ${ }^{27}$ Additionally, the island in the lower left corner, which is attached to a substrate step edge, changed in shape while scanning. Interestingly, upon reshaping, the islands tend to form straight step edges. When comparing the shape of this island in Fig.
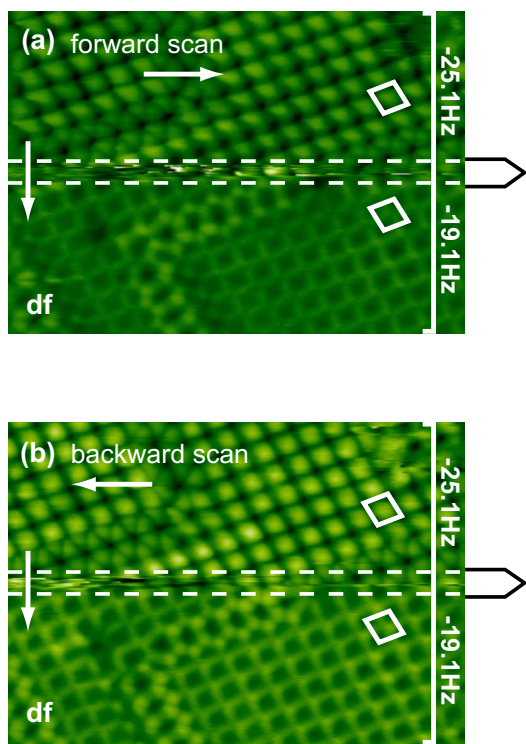
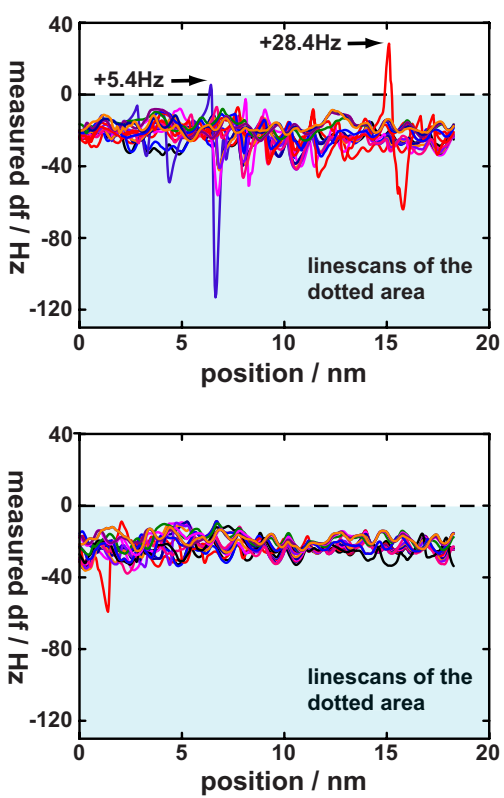

FIG. 2. (Color online) Forward (a) and backward scan (b) and the frequency shift channel. In the upper part, the frequency shift setpoint was set to $-25.1 \mathrm{~Hz}$ as indicated in the images. After the manipulation happened in the area enclosed by dotted lines, the frequency shift was set to $-19.1 \mathrm{~Hz}$ for stable imaging. Analyzing the area where manipulation was achieved reveals two line scans in the forward scan with a positive frequency shift, which is related to a repulsive interaction between tip and molecules (see Ref. 33). 

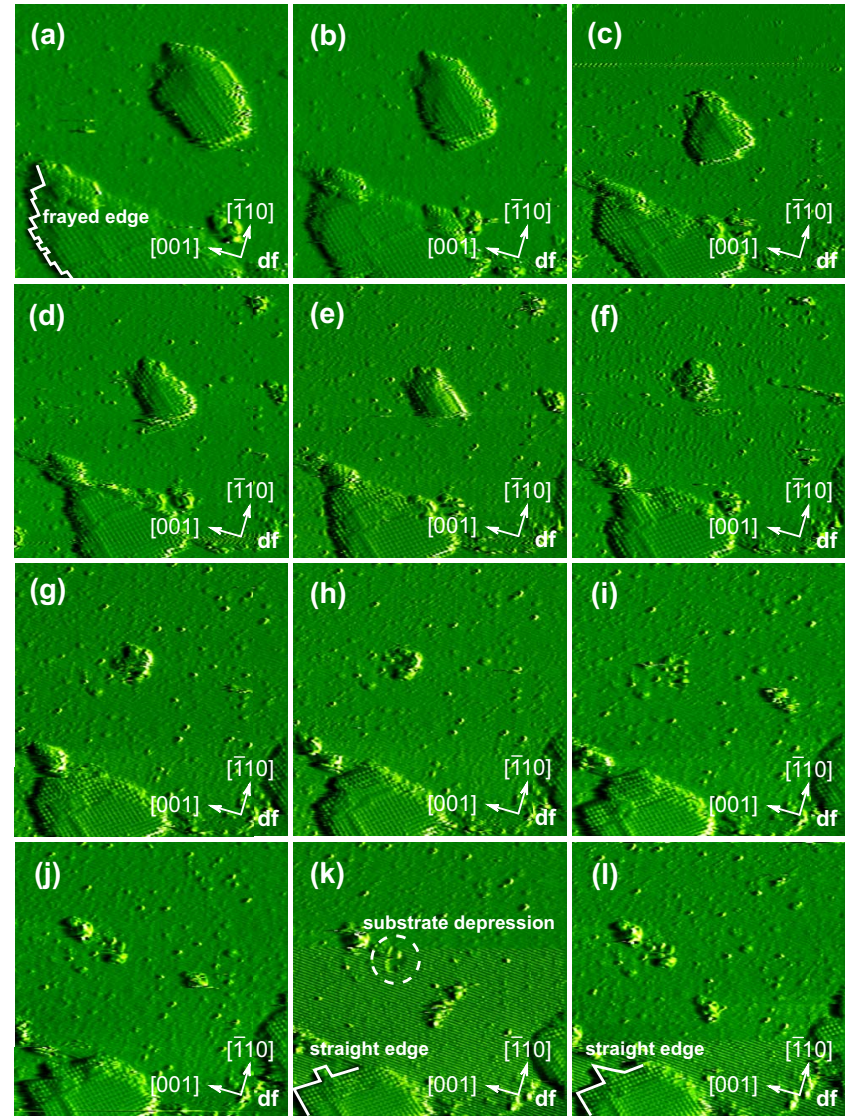

FIG. 3. (Color online) Series of NC-AFM images at almost the same frequency shift setpoint $(13.0-15.0 \mathrm{~Hz}), 62 \times 62 \mathrm{~nm}^{2}$, frequency shift channel, and backward scans. The first image is taken approximately $100 \mathrm{~min}$ after molecule deposition. All following images are taken successively with a time per frame of $9 \mathrm{~min} 20 \mathrm{~s}$. The isolated island in the upper part of the image shrinks in size during scanning until it completely vanishes. In image (k) a substrate depression is observed, which is assumed to represent the nucleation seed for the island growth (see Ref. 27).

3(a) with Fig. 3(k) or Fig. 3(1), the initially frayed edges become straight. This indicates that straight edges are more stable than corrugated edges, which can be explained by the increased coordination number of the $\mathrm{C}_{60}$ molecules forming the edges. For a straight edge, an edge $\mathrm{C}_{60}$ molecule has three neighbors while more corrugated edges would result in a smaller coordination number. The $\mathrm{C}_{60}$ molecules that are detached from the island are observed to move to nearby step edges or $\mathrm{C}_{60}$ islands in the vicinity. Moreover, they might be anchored to surface defects, which could explain the increase in single bright protrusions seen in this image series.

We analyzed the frequency shift data taken during the manipulation process shown in Fig. 3. No significant manipulation events could be identified here. In particular, we do not observe positive frequency shift values. Thus, despite the fact that clear tip-induced repulsive manipulation processes exist as demonstrated in Fig. 2, the mechanism of manipulation in Fig. 3 is not as clear. Consequently, a thermally assisted manipulation ${ }^{23}$ is not excluded in this case.

In conclusion, almost quadratic pits were created in ordered $\mathrm{C}_{60}$ islands using $\mathrm{NC}-\mathrm{AFM}$ at room temperature. Upon gradually approaching the tip toward the surface, the interaction strength of the NC-AFM tip with the $\mathrm{C}_{60}$ molecules can be increased until manipulation is induced. Analyzing the frequency shift curves during manipulation allows for determining the manipulation mode and unambiguously as- signing the observed manipulation to repulsive tip-sample interaction. Retracting the tip after manipulation allows for reproducible imaging and evaluating the manipulation result. Besides hole formation, we also observed the movement of a domain boundary by one repeat distance. This movement is associated with the manipulation of a stacking fault and a rather large mass transport. Additionally, we observed reshaping and removal of islands. For the latter manipulation it is not clear so far, whether it might be induced by thermally assisted manipulation.

Financial support from the Deutsche Forschungsgemeinschaft (DFG) through an Emmy Noether grant (KU 1980/ 1-2) is gratefully acknowledged.

${ }^{1}$ C. Joachim, J. K. Gimzewski, and A. Aviram, Nature (London) 408, 541 (2000).

${ }^{2}$ G. E. Moore, Electronics 38, 114 (1965).

${ }^{3}$ J. M. Mativetsky, S. A. Burke, S. Fostner, and P. Grütter, Small 3, 818 (2007).

${ }^{4}$ J. M. Mativetsky, S. A. Burke, S. Fostner, and P. Grütter, Nanotechnology 18, 105303 (2007).

${ }^{5}$ G. M. Whitesides and B. Grzybowski, Science 295, 2418 (2002).

${ }^{6}$ J. V. Barth, G. Costantini, and K. Kern, Nature (London) 437, 671 (2005).

${ }^{7}$ A. Kühnle, Curr. Opin. Colloid Interface Sci. 14, 157 (2009).

${ }^{8}$ T. Junno, K. Deppert, L. Montelius, and L. Samuelson, Appl. Phys. Lett. 66, 3627 (1995).

${ }^{9}$ D. Eigler and E. Schweizer, Nature (London) 344, 524 (1990).

${ }^{10}$ L. Bartels, G. Meyer, and K. H. Rieder, Phys. Rev. Lett. 79, 697 (1997)

${ }^{11}$ G. Meyer, J. Repp, S. Zöphel, K.-F. Braun, S. W. Hla, S. Fölsch, L. Bartels, F. Moresco, and K. H. Rieder, Single Mol. 1, 79 (2000).

${ }^{12}$ A. J. Heinrich, C. P. Lutz, J. A. Gupta, and D. M. Eigler, Science 298, 1381 (2002).

${ }^{13}$ N. Martsinovich, L. Kantorovich, R. H. J. Fawcett, M. J. Humphry, and P. H. Beton, Small 4, 765 (2008)

${ }^{14}$ G. Binnig, C. Quate, and C. Gerber, Phys. Rev. Lett. 56, 930 (1986)

${ }^{15}$ Y. Sugimoto, M. Abe, S. Hirayama, N. Oyabu, O. Custance, and S. Morita, Nature Mater. 4, 156 (2005).

${ }^{16}$ Y. Sugimoto, P. Pou, O. Custance, P. Jelinek, M. Abe, R. Pérez, and S. Morita, Science 322, 413 (2008).

${ }^{17}$ Y. Sugimoto, P. Jelinek, P. Pou, M. Abe, S. Morita, R. Pérez, and O. Custance, Phys. Rev. Lett. 98, 106104 (2007).

${ }^{18}$ S. Morita, Y. Sugimoto, N. Oyabu, R. Nishi, O. Custance, Y. Sugawara and M. Abe, J. Electron Microsc. 53, 163 (2004).

${ }^{19}$ M. Ternes, C. P. Lutz, C. F. Hirjibehedin, F. J. Giessibl, and A. J. Heinrich, Science 319, 1066 (2008).

${ }^{20}$ S. Hirth, F. Ostendorf, and M. Reichling, Nanotechnology 17, S148 (2006).

${ }^{21}$ R. Nishi, D. Miyagawa, Y. Seino, I. Yi, and S. Morita, Nanotechnology 17, S142 (2006).

${ }^{22}$ S. Kawai and H. Kawakatsu, Appl. Phys. Lett. 89, 023113 (2006).

${ }^{23}$ N. Martsinovich and L. Kantorovich, Nanotechnology 20, 135706 (2009).

${ }^{24}$ T. Trevethan, M. Watkins, L. N. Kantorovich, A. L. Shluger, J. PoleselMaris, and S. Gauthier, Nanotechnology 17, 5866 (2006).

${ }^{25}$ T. Trevethan, L. Kantorovich, J. Polesel-Maris, S. Gauthier, and A. Shluger, Phys. Rev. B 76, 085414 (2007).

${ }^{26}$ N. Oyabu, Y. Sugimoto, M. Abe, O. Custance, and S. Morita, Nanotechnology 16, S112 (2005).

${ }^{27}$ F. Loske, R. Bechstein, J. Schütte, F. Ostendorf, M. Reichling, and A. Kühnle, Nanotechnology 20, 065606 (2009).

${ }^{28}$ F. Loske, P. Rahe, and A. Kühnle, Nanotechnology 20, 264010 (2009).

${ }^{29}$ H. W. Kroto, J. R. Heath, S. C. O'Brien, R. F. Curl, and R. E. Smalley, Nature (London) 318, 162 (1985).

${ }^{30}$ C. Joachim, J. K. Gimzewsky, R. R. Schlittler, and C. Chavy, Phys. Rev. Lett. 74, 2102 (1995)

${ }^{31}$ C. Joachim and J. K. Gimzewski, Chem. Phys. Lett. 265, 353 (1997).

${ }^{32}$ C. Joachim, J. K. Gimzewski, and H. Tang, Phys. Rev. B 58, 16407 (1998).

${ }^{33}$ P. Rahe, R. Bechstein, J. Schütte, F. Ostendorf, and A. Kühnle, Phys. Rev. B 77, 195410 (2008).

${ }^{34}$ J. Schütte, R. Bechstein, P. Rahe, M. Rohlfing, A. Kühnle, and H. Langhals, Phys. Rev. B 79, 045428 (2009). 